\title{
Limits on stable iron in Type la supernovae from near-infrared spectroscopy ${ }^{\star}$
}

\author{
A. Flörs ${ }^{1,2,3}$, J. Spyromilio ${ }^{1}$, K. Maguire ${ }^{4}$, S. Taubenberger ${ }^{1,2}$, W. E. Kerzendorf ${ }^{1}$, and S. Dhawan ${ }^{5}$ \\ ${ }^{1}$ European Southern Observatory, Karl-Schwarzschild-Straße 2, 85748 Garching bei München, Germany \\ e-mail: afloers@eso.org \\ ${ }^{2}$ Max-Planck-Institut für Astrophysik, Karl-Schwarzschild-Straße 1, 85748 Garching bei München, Germany \\ ${ }^{3}$ Physik-Department, Technische Universität München, James-Franck-Straße 1, 85748 Garching bei München, Germany \\ ${ }^{4}$ Astrophysics Research Centre, School of Mathematics and Physics, Queen's University Belfast, Belfast BT7 1NN, UK \\ 5 Oskar Klein Centre, Department of Physics, Stockholm University, SE 10691 Stockholm, Sweden
}

Received 28 May 2018 / Accepted 10 October 2018

\begin{abstract}
We obtained optical and near infrared spectra of Type Ia supernovae (SNe Ia) at epochs ranging from 224 to 496 days after the explosion. The spectra show emission lines from forbidden transitions of singly ionised iron and cobalt atoms. We used non-local thermodynamic equilibrium (NLTE) modelling of the first and second ionisation stages of iron, nickel, and cobalt to fit the spectra using a sampling algorithm allowing us to probe a broad parameter space. We derive velocity shifts, line widths, and abundance ratios for iron and cobalt. The measured line widths and velocity shifts of the singly ionised ions suggest a shared emitting region. Our data are fully compatible with radioactive ${ }^{56} \mathrm{Ni}$ decay as the origin for cobalt and iron. We compare the measured abundance ratios of iron and cobalt to theoretical predictions of various SN Ia explosion models. These models include, in addition to ${ }^{56} \mathrm{Ni}$, different amounts of ${ }^{57} \mathrm{Ni}$ and stable ${ }^{54,56} \mathrm{Fe}$. We can exclude models that produced only ${ }^{54,56} \mathrm{Fe}$ or only ${ }^{57} \mathrm{Ni}$ in addition to ${ }^{56} \mathrm{Ni}$. If we consider a model that has ${ }^{56} \mathrm{Ni}$, ${ }^{57} \mathrm{Ni}$, and ${ }^{54,56} \mathrm{Fe}$ then our data imply that these ratios are ${ }^{54,56} \mathrm{Fe} /{ }^{56} \mathrm{Ni}=0.272 \pm 0.086$ and ${ }^{57} \mathrm{Ni} /{ }^{56} \mathrm{Ni}=0.032 \pm 0.011$.
\end{abstract}

Key words. supernovae: general - line: formation - line: identification - radiation mechanisms: thermal

\section{Introduction}

Type Ia supernovae (SNe Ia) are a remarkably uniform class of objects. Exceptions such as overly bright (e.g. SN 1991T Filippenko et al. 1992b; Phillips et al. 1992; Ruiz-Lapuente et al. 1992) or overly faint (e.g. SN 1991bg Filippenko et al. 1992a; Leibundgut et al. 1993; Turatto et al. 1996) supernovae have been extensively studied (see Taubenberger 2017, for a recent overview of the various Type Ia subtypes). High-cadence all sky surveys (e.g. Kulkarni 2012; Shanks et al. 2015; Chambers et al. 2016; Kochanek et al. 2017) have discovered that members of the class exhibit a broad spectrum of disorders. However, the Branch-normal Type Ias (Branch et al. 2006) remain the dominant detected class and one of the best distance indicators at the disposal of astronomers. Following calibration procedures, for example, the one by Phillips (1993), that correlate the width of the light curve with the peak brightness, SNe Ia exhibit a very small dispersion in their absolute magnitudes. As such, their use in cosmology is extensive and understanding them is of general interest (Leibundgut \& Sullivan 2018).

Colgate \& McKee (1969) proposed powering of the optical and infrared (IR) displays of SNe to originate in energy deposition from the decay of radioactive ${ }^{56} \mathrm{Ni}$ to ${ }^{56} \mathrm{Co}$ and subsequently to ${ }^{56} \mathrm{Fe}$. Depending on the temperature and density, burning to nuclear statistical equilibrium converts much of the progenitor white dwarf to iron group elements (Hoyle \& Fowler 1960; see

\footnotetext{
* Based on observations collected at the La Silla and Paranal observatories of the European Southern Observatory, Chile in time allocated to proposals 63.H-0527, 63.H-0649 and 089.D-0647(A), and at the Gemini North observatory under proposal GN-2015A-FT-3.
}

Seitenzahl et al. 2013 for a recent calculation of yields). Many studies have provided both direct and indirect evidence for this scenario. Direct evidence can be found in Churazov et al. (2014) who detected $\gamma$-ray lines from the decay of ${ }^{56} \mathrm{Co}$ at 847 and $1238 \mathrm{keV}$. Indirect evidence was shown by Kuchner et al. (1994) who, in Type Ia late-time optical spectra $\approx 200$ days, observed that the doubly ionised emission lines of $\mathrm{Co}$ and Fe evolved according to the expected ratio that would result from the production of $\mathrm{Fe}$ as the daughter product of the radioactive decay of ${ }^{56} \mathrm{Co}$. The total energy of the explosion maps onto the mass of nucleosynthesised ${ }^{56} \mathrm{Ni}$ and the luminosity at maximum light is directly linked to the mass of ${ }^{56} \mathrm{Ni}$ (Arnett 1982). The determination of the mass of ${ }^{56} \mathrm{Ni}$ based on optical and IR photometry and spectroscopy has been the subject of many papers and almost as many models. In general, of order $0.4 M_{\odot}-0.8 M_{\odot}$ of ${ }^{56} \mathrm{Ni}$ is found to be produced in Branch-normal SNe Ia (Arnett 1982; for a more recent study see Childress et al. 2015).

The post maximum optical and near-infrared (NIR) spectra of SNe Ia exhibit a plethora of iron group emission lines, predominantly in the singly and doubly ionised states (Axelrod 1980; Fransson \& Chevalier 1989; Kozma et al. 2005; Fransson \& Jerkstrand 2015). Many authors have provided an extensive analysis of the physical conditions in the ejecta and the processes that generate the spectrum. Post-maximum spectra have been published by many authors and show remarkable similarity in features and evolution (Mazzali et al. 1998). Maeda et al. (2010) made the startling discovery that often the singly ionised emission lines of the iron group elements exhibit a different systemic velocity when compared to their doubly ionised counterparts and proposed that there exists a region within the 
Table 1. Overview of spectra in our sample.

\begin{tabular}{|c|c|c|c|c|c|c|c|c|}
\hline Supernova & $\begin{array}{c}\mathrm{E}(B-V)^{a} \\
(\mathrm{mag})\end{array}$ & $\mathrm{z}^{b}$ & Date of max. & Epoch $^{c}$ & Telescope & Instrument & $\begin{array}{c}\text { Resolution } \\
\lambda / \Delta \lambda\end{array}$ & Reference \\
\hline \multirow[t]{2}{*}{ SN 1998bu } & $0.30^{d}$ & 0.002992 & 1998 May 21 & $269 d$ & NTT & SOFI & 500 & $1^{e}$ \\
\hline & & & & $364 d$ & VLT & ISAAC, FORS1 & 1500,440 & $1^{e}$ \\
\hline SN 2012cg & $0.20^{f}$ & 0.001458 & 2012 June 03 & $357 \mathrm{~d}$ & VLT & X-shooter & $6200 / 8800 / 5300^{g}$ & 2 \\
\hline SN 2012fr & 0.018 & 0.005457 & 2012 Nov 12 & $375 d$ & VLT & X-shooter & $6200 / 8800 / 5300^{g}$ & 2 \\
\hline \multirow{2}{*}{ SN 2013aa } & 0.169 & 0.003999 & 2013 Feb 21 & $378 d$ & VLT & X-shooter & $6200 / 8800 / 5300^{g}$ & 2 \\
\hline & & & & $443 d$ & VLT & X-shooter & $6200 / 8800 / 5300^{g}$ & 3 \\
\hline SN 2013cs & 0.082 & 0.009243 & 2013 May 26 & $321 \mathrm{~d}$ & VLT & X-shooter & $6200 / 8800 / 5300^{g}$ & 2 \\
\hline SN 2013ct & 0.025 & 0.003843 & 2013 May 04 & $247 d$ & VLT & X-shooter & $6200 / 8800 / 5300^{g}$ & 2 \\
\hline \multirow[t]{2}{*}{ SN 2014J } & $1.37^{h}$ & 0.000677 & 2014 Feb 01 & $468 d$ & Gemini-North & GNIRS & 1800 & 4 \\
\hline & & & & $496 \mathrm{~d}$ & Gemini-North & GNIRS & 1800 & 4 \\
\hline PSN J1149 & 0.025 & 0.005589 & 2015 July 12 & $224 d$ & VLT & X-shooter & $6200 / 8800 / 5300^{e}$ & 3 \\
\hline
\end{tabular}

Notes. ${ }^{(a)} \mathrm{MW} \mathrm{E}(B-V)$ in magnitudes from Schlafly \& Finkbeiner (2011). If additional host galaxy extinction is present we quote the combined Galactic and host galaxy $\mathrm{E}(B-V)$ in magnitudes. ${ }^{(b)}$ Heliocentric redshifts are taken from the Nasa Extragalactic Database (NED). ${ }^{(c)}$ Days after the explosion, assuming a rise time of $\sim 18$ days (Ganeshalingam et al. 2011). The spectra were scaled to match the photometry at this phase. ${ }^{(d)}$ Jha et al. (1999) report the extinction towards the $\mathrm{SN}$ as $A_{V}=0.94 \mathrm{mag} .{ }^{(e)} \mathrm{We}$ subtracted a light echo as found by Cappellaro et al. (2001) scaled up by a factor of two (see Spyromilio et al. 2004). ${ }^{(f)}$ Silverman et al. (2012) found host galaxy extinction of $\mathrm{E}(B-V)=0.18 \mathrm{mag} .{ }^{(g)} \mathrm{Resolution}$ of the three X-shooter arms UVB/VIS/NIR ${ }^{(h)}$ Amanullah et al. $(2014)$ determine $\mathrm{E}(B-V)=1.37$ mag with low total-to-selective extinction $\mathrm{R}_{V}=1.4$. References. (1) Spyromilio et al. (2004); (2) Maguire et al. (2016); (3) Maguire et al. (2018); (4) Dhawan et al. (2018).

core of the ejecta where electron capture processes dominate the nucleosynthetic yield.

In this paper, we fit late-time NIR observations with emission lines from NLTE level populations to derive the evolution of the mass ratio of Co II to Fe II. We aim to place limits on the stable Fe and ${ }^{57} \mathrm{Ni}$ in the ejecta by observing the evolution in the mass ratio of Co to Fe. In Sect. 2, we briefly describe the observations and in Sect. 3, we present our NLTE emission line fit code. In Sect. 4, we present the results of our spectral fits and derive the mass ratio $M_{\mathrm{Co} \text { II }} / M_{\mathrm{Fe} \text { II }}$. In Sect. 5, we compare the mass ratio results of our SN sample with explosion model predictions within the Bayesian framework. The results are discussed in Sect. 6.

\section{Observations}

Four sets of data are included in this work (see Table 1). One set from the GNIRS instrument on Gemini-North, two from the X-shooter instrument at ESO's Paranal observatory and the fourth from SOFI at the New Technology Telescope (NTT) and ISAAC+FORS1 at the Very Large Telescope (VLT). GNIRS, SOFI, and ISAAC cover the NIR bands, FORS1 covers the optical while X-shooter covers both the optical and NIR bands. As we are mainly interested in the abundances of various radioactive isotopes all epochs in this work are given in days after the explosion, assuming a rise time of $\sim 18$ days (Ganeshalingam et al. 2011).

We use two NIR spectra of SN2014J obtained with GNIRS at Gemini-North (see Dhawan et al. 2018). Optical+NIR spectra of SN 2013cg, SN 2012fr, SN 2013aa, SN 2013cs and SN 2013ct were obtained by Maguire et al. (2016) at the VLT. Maguire et al. (2018) also obtained a spectrum of PSNJ11492548-0507138 (hereafter PSNJ1149) and a second spectrum of SN 2013aa. We also use two spectra of SN 1998bu (Spyromilio et al. 2004). For details of the reduction see the publication papers.

A number of broad $\left(\approx 7000-9000 \mathrm{~km} \mathrm{~s}^{-1}\right)$ emission lines at 1.55 and $1.65 \mu \mathrm{m}$ are evident in the data. We identify these as emission by the $1.644 \mu \mathrm{m}$ [Fe II] line arising from the $\mathrm{a}^{4} \mathrm{~F}$ $\mathrm{a}^{4} \mathrm{D}$ multiplet and the $1.547 \mu \mathrm{m}$ [Co II] line from the $\mathrm{a}^{5} \mathrm{~F}-\mathrm{b}^{3} \mathrm{~F}$ multiplet. The $\mathrm{a}^{4} \mathrm{~F}-\mathrm{a}^{4} \mathrm{D}$ [Fe II] multiplet includes the $1.533 \mu \mathrm{m}$
[Fe II] line. The identification of the various features has been extensively covered in the literature. The detection of the $1.257 \mu \mathrm{m} \mathrm{a}{ }^{6} \mathrm{D}_{9 / 2}-\mathrm{a}^{4} \mathrm{D}_{7 / 2}$ confirms the identification and strength of the $\mathrm{a}^{4} \mathrm{~F}_{9 / 2}-\mathrm{a}^{4} \mathrm{D}_{7 / 2} 1.644 \mu \mathrm{m}$ line as they arise from the same upper level. Similarly, the detection in many of our spectra of the $1.090 \mu \mathrm{m}\left(\mathrm{a}^{3} \mathrm{~F}_{4}-\mathrm{b}^{3} \mathrm{~F}_{4}\right)$ line of [Co II] which shares an upper level with the $1.547 \mu \mathrm{m}\left(\mathrm{a}^{5} \mathrm{~F}_{4}-\mathrm{b}^{3} \mathrm{~F}_{4}\right)$ line also secures the identification.

\section{Fitting the spectra}

\subsection{Emission line model}

We use a one zone model with Fe II, Ni II, and Co II; it also includes Co III if the spectrum covers the $6000 \AA$ region. For this set of ions, we solve the NLTE rate equations treating only collisional excitation and de-excitation by collisional and radiative processes to compute energy level populations and derive forbidden line emissivities. For the collisional processes we use a thermal electron gas characterised by a Boltzmann distribution and an electron density. We ignore radiative transfer effects as the optical depths of the lines under consideration are very low at the observed epochs. We also do not consider non-thermal excitations as the energy going into this channel at the relatively high electron densities we determine is also very low (Fransson \& Chevalier 1989). We do not include charge exchange and time-dependent terms in the NLTE rate equations. Even though we do not treat continuum processes directly, we include a parametrized smooth continuum in the model (see Sect. 3.2). We use such a smooth continuum to include uncertainties due to true continuum processes in the early spectra and potentially low-level light echoes. We do not treat energy deposition in the ejecta, and as a result our model does not match the emitted radiation over all wavelengths, but only for certain lines. We do not solve the ionisation balance but treat the number of emitting ions as a fit parameter. Effectively, we solve a simplified and parametrized NLTE problem.

A one-zone model, by default spherically symmetric, convolved with a Gaussian line profile serves to fit the spectra and 
extract the salient properties of the lines (flux, rest velocity, and Doppler broadening). One evident limitation of the onezone model is that different ionisation stages, not necessarily co-located in the ejecta (see Sect. 4.2), are likely to have different excitation conditions in some fraction of the emitting regions. This work, which concentrates on the singly ionised species, is not significantly affected by this limitation.

The features at 4700 and $5200 \AA$ are a blend of singly and doubly ionised iron, with possibly asymmetric line profiles. Changes of the velocity offset and width of the $4700 \AA$ feature with time suggest that this wavelength region is not optically thin until 400 days after the explosion (Black et al. 2016). A detailed analysis of this feature would require a more sophisticated model process. As [Fe III] only slightly influences the rest of the Optical+NIR spectrum we do not attempt to fit spectral features below $5500 \AA$.

We fit the $5900 \AA$ A region with [Co III] and the double-peaked feature between 7000 and $7800 \AA$ with [Fe II]+[Ni II]. All spectra exhibit a strong emission feature between $8500 \AA$ and $9900 \AA$. We can reproduce the feature at $8600 \AA$ with lines from the Fe II $\mathrm{a}^{4} \mathrm{~F}-\mathrm{a}^{4} \mathrm{P}$ and $\mathrm{Co}$ II $\mathrm{a}^{3} \mathrm{~F}-\mathrm{b}^{3} \mathrm{~F}$ multiplets, but in spectra which are less than one year old the red part of the feature between 8800 and $9900 \AA$ cannot be well explained by emission from only ions in our sample. Models by Botyánszki \& Kasen (2017), among other authors, suggest the presence of S III in this region. We only use the $8600 \AA$ feature for spectra of SN 2014J, but we do not use the 8800-9900 ̊̊region.

The strongest lines of the considered ions are given in Table 2. An overview of the model ions with their respective atomic data is given in Table 3. For Fe II we use the atomic data from Bautista et al. (2015) as they provide collision strengths for the higher levels responsible for optical transitions. The spectra are displayed in Fig. 1 in chronological order. The data are corrected for the redshift of the host, as well as Galactic, and, if applicable, host galaxy extinction according to Table 1. The simple model of Fe II, Co II, Co III and Ni II fits the data well above $5500 \AA$.

\subsection{Parameter estimation}

We explore the parameter space using the nested-sampling algorithm Nestle ${ }^{1}$ (Shaw et al. 2007). The algorithm allows us to sample from our flat priors over a large range by optimising the selection of variables based on the quality of the earlier fits. To compare the model with the data we assume a $\chi^{2}$ likelihood. Our set of four ions shares the same temperature and electron density. Each ion is allowed to have its own line width, velocity offset, and strength. Uniform priors are used for all parameters except the electron density, for which we use a log-uniform distribution. There exists a significant body of work in the literature starting with Axelrod (1980), and a plethora of other authors (see e.g. Kuchner et al. 1994; Kozma et al. 2005; Fransson \& Jerkstrand 2015; Botyánszki \& Kasen 2017) that have shown that temperatures for the ejecta of a SNe Ia during the first year after explosion lie in the range of 2000-15000 K. For our modelling we adopt the following boundaries for the priors: temperature range $(2000-15000 \mathrm{~K})$, electron densities between $10^{4}$ and $10^{7} \mathrm{~cm}^{-3}$, Doppler widths between 2 and $15 \times 10^{3} \mathrm{~km} \mathrm{~s}^{-1}$, and shifts between -3 and $3 \times 10^{3} \mathrm{~km} \mathrm{~s}^{-1}$. The very large allowed range for the temperature prior does not affect the resulting fit.

1 https://github.com/kbarbary/nestle
Table 2. Strongest lines of the included ions in the optical and NIR.

\begin{tabular}{|c|c|c|}
\hline$\underline{\lambda_{\text {rest }}(\mu \mathrm{m})}$ & Ion & Transition \\
\hline 0.5528 & {$[\mathrm{Fe} \mathrm{II}]$} & $a^{4} F_{7 / 2}-a^{2} D_{5 / 2}$ \\
\hline 0.5888 & [Co III] & $a^{4} F_{9 / 2}-a^{2} G_{9 / 2}$ \\
\hline 0.5908 & [Co III] & $a^{4} F_{7 / 2}-a^{2} G_{7 / 2}$ \\
\hline 0.6197 & [Co III] & $a^{4} F_{7 / 2}-a^{2} G_{9 / 2}$ \\
\hline 0.6578 & [Co III] & $a^{4} F_{9 / 2}-a^{4} P_{5 / 2}$ \\
\hline 0.6855 & [Co III] & $a^{4} F_{7 / 2}-a^{4} P_{3 / 2}$ \\
\hline 0.7155 & {$[\mathrm{Fe} \mathrm{II}]$} & $a^{4} F_{9 / 2}-a^{2} G_{9 / 2}$ \\
\hline 0.7172 & {$[\mathrm{Fe} \mathrm{II}]$} & $a^{4} F_{7 / 2}-a^{2} G_{7 / 2}$ \\
\hline 0.7378 & [Ni II] & $\mathrm{z}^{2} \mathrm{D}_{5 / 2}-\mathrm{a}^{2} \mathrm{~F}_{7 / 2}$ \\
\hline 0.7388 & [Fe II] & $a^{4} F_{5 / 2}-a^{2} G_{7 / 2}$ \\
\hline 0.7414 & [Ni II] & $\mathrm{z}^{2} \mathrm{D}_{3 / 2}-\mathrm{a}^{2} \mathrm{~F}_{5 / 2}$ \\
\hline 0.7453 & {$[\mathrm{Fe} \mathrm{II}]$} & $a^{4} F_{7 / 2}-a^{2} G_{9 / 2}$ \\
\hline 0.7638 & {$[\mathrm{Fe} \mathrm{II}]$} & $a^{6} D_{7 / 2}-a^{4} P_{5 / 2}$ \\
\hline 0.7687 & {$[\mathrm{Fe} \mathrm{II}]$} & $a^{6} D_{5 / 2}-a^{4} P_{3 / 2}$ \\
\hline 0.8617 & {$[\mathrm{Fe} \mathrm{II}]$} & $a^{4} F_{9 / 2}-a^{4} P_{5 / 2}$ \\
\hline 0.9345 & [Co II $]$ & $a^{3} F_{3}-a^{1} D_{2}$ \\
\hline 1.0190 & [Co II $]$ & $a^{3} F_{4}-b^{3} F_{4}$ \\
\hline 1.0248 & [Co II $]$ & $a^{3} F_{3}-b^{3} F_{3}$ \\
\hline 1.2570 & {$[\mathrm{Fe} \mathrm{II}]$} & $a^{6} D_{9 / 2}-a^{4} D_{7 / 2}$ \\
\hline 1.2943 & {$[\mathrm{Fe} \mathrm{II}]$} & $a^{6} D_{5 / 2}-a^{4} D_{5 / 2}$ \\
\hline 1.3206 & [Fe II] & $a^{6} D_{7 / 2}-a^{4} D_{7 / 2}$ \\
\hline 1.5335 & [Fe II] & $a^{4} F_{9 / 2}-a^{4} D_{5 / 2}$ \\
\hline 1.5474 & [Co II $]$ & $a^{5} F_{5}-b^{3} F_{4}$ \\
\hline 1.5488 & [Co III] & $\mathrm{a}^{2} \mathrm{G}_{9 / 2}-\mathrm{a}^{2} \mathrm{H}_{9 / 2}$ \\
\hline 1.5995 & {$[\mathrm{Fe} \mathrm{II}]$} & $a^{4} F_{7 / 2}-a^{4} D_{3 / 2}$ \\
\hline 1.6440 & [Fe II] & $a^{4} F_{9 / 2}-a^{4} D_{7 / 2}$ \\
\hline
\end{tabular}

Table 3. Ions included in the fits and their atomic data sets.

\begin{tabular}{cccc}
\hline \hline Ion & Levels $^{a}$ & Ref. $A_{i j}{ }^{b}$ & Ref. $\Upsilon_{i j}^{c}$ \\
\hline Fe II & 52 & 1 & 1 \\
Co II & 15 & 2 & 2 \\
Co III & 15 & 3 & 3 \\
Ni II & 18 & 4 & 5 \\
\hline
\end{tabular}

Notes. ${ }^{(a)}$ Energy levels and statistical weights are taken from NIST (Kramida et al. 2018). ${ }^{(b)}$ Einstein $A$ coefficient between levels $i$ and $j$. ${ }^{(c)}$ Maxwellian averaged collisional strength between levels $i$ and $j$.

References. (1) Bautista et al. (2015); (2) Storey et al. (2016); (3) Storey $\&$ Sochi (2016); (4) Cassidy et al. (2016); (5) Cassidy et al. (2010)

We note the presence of a weak continuum in some spectra bluewards of $9500 \AA$; in those cases, we subtract a linear background (grey bands in Fig. 1) in the optical. Widths and shifts of individual ions are determined using a global fit where the individual species are allowed to vary within the prior space.

Besides the fit parameters noted above, the fits provide us with the individual emission line emissivities. The ratios of the transitions arising from within the $\mathrm{a}^{4} \mathrm{~F}-\mathrm{a}^{4} \mathrm{D}$ Fe II multiplet show little variation with temperature as the difference in the energy levels within $\mathrm{a}^{4} \mathrm{D}$ is less than $1000 \mathrm{~cm}^{-1}$ and for the strongest lines at $1.644 \mu \mathrm{m}$ and $1.533 \mu \mathrm{m}$ less than $500 \mathrm{~cm}^{-1}$. The higherlying states responsible for lines in the optical vary significantly in strength over the considered temperature range and thus allow us to estimate the electron temperature. However, there is a degeneracy between electron density and temperature. Good fits 

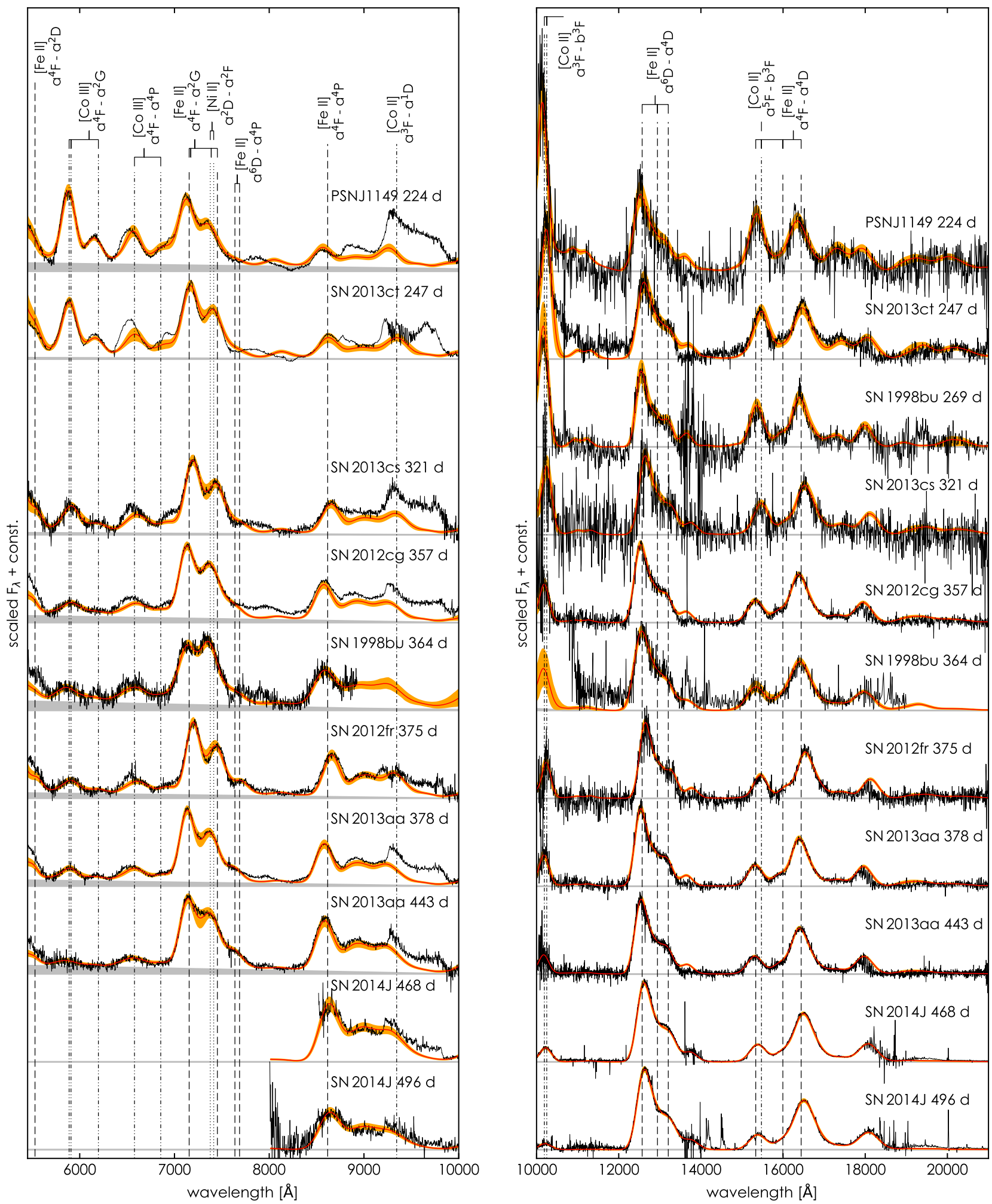

Fig. 1. Optical (left panel) and NIR (right panel) spectra of SNe Ia obtained with X-shooter, FORS1, and ISAAC at the VLT, SOFI at the NTT, and GNIRS at Gemini-North. The spectra are arranged in epoch starting with the youngest at the top and corrected for redshift and extinction. Fluxes are normalized to the $7300 \AA$ [Fe II] + [Ni II] feature (optical) and $12600 \AA$ [Fe II] feature (NIR). The red line indicates the mean flux of all fit models at each wavelength, the orange shaded area marks the $68 \%$ uncertainty of the fit. Dashed vertical lines indicate the strongest lines as

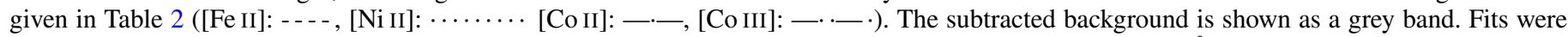
performed for the optical and NIR spectra at the same time. The lines/ions composing the features below $5500 \AA$ are not included in our fits.

can be achieved with high temperature and low densities, and vice versa. Some of these high temperatures and low densities would not be compatible with a prior of $\sim 0.4-0.8 M_{\odot}$ of iron group elements. We have not adopted such a prior for the fitting as absolute mass estimates suffer considerably from systematics (e.g. distance). We want to emphasise that the uncertainties from this degeneracy are much smaller than the priors (see Table 4). 


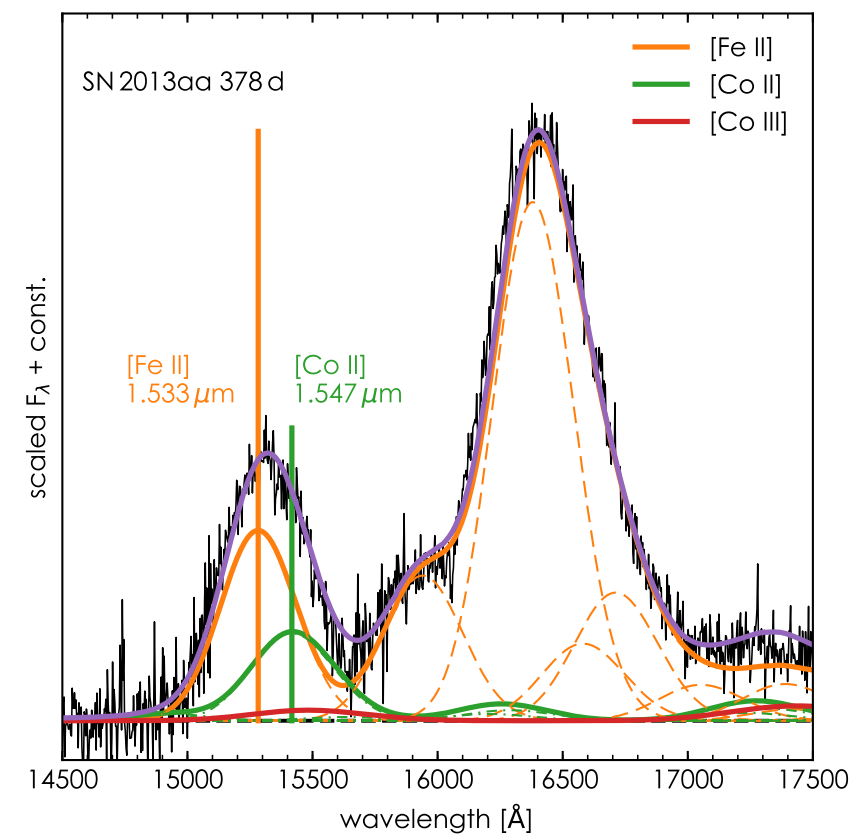

Fig. 2. Best fit model of SN 2013aa at $378 \mathrm{~d}$ in the $H$-band. Shown is the extinction and redshift corrected spectrum (black line) and individual contributions from the ions Fe II (orange), Co II (green) and Co III (red) as solid curves. The purple line is the combined emission of the three ions. The two solid vertical lines are used to compute $M_{\mathrm{Co} \mathrm{II}} / M_{\mathrm{Fe} \mathrm{II}}$. Dashed curves indicate the contribution of the individual lines in the blended region. We do not show Ni II as it does not have any lines in this region.

\section{Fit results}

\subsection{Fit parameters and $M_{\mathrm{Co} \|} / M_{\mathrm{Fe} I I}$}

The ratio of the $H$-band [Co II] to [Fe II] lines places limits on the temperature of the ejecta of above $1500 \mathrm{~K}$. Below this temperature, the blue wing of the $1.55 \mu \mathrm{m}$ spectral feature is underproduced and the blend of the 1.634 [Co II] and 1.644 [Fe II] lines which accounts for the $1.65-\mu \mathrm{m}$ feature is unable to reproduce the spectra. The presence of the shoulder from the $\mathrm{a}^{4} \mathrm{~F}_{7 / 2}-\mathrm{a}^{4} \mathrm{D}_{3 / 2}$ [Fe II] line at $1.599 \mu \mathrm{m}$ in spectra at all epochs indicates that the emission originates from a high-density region $\left(n_{\mathrm{e}}>10^{5} \mathrm{~cm}^{-3}\right)$.

The absolute mass of the emitting material strongly depends on the assumed temperature in the ejecta and the distance to the object. We do not consider that our model is sophisticated enough to provide detailed constraints on these parameters (see for example Fransson \& Jerkstrand 2015 or Diamond et al. 2018 for such work). On the other hand, mass ratios are only weakly dependent on the temperature. In particular, from the line ratio of $1.547 \mu \mathrm{m}$ [Co II] to $1.533 \mu \mathrm{m}$ [Fe II] (see Fig. 2) we can determine the mass fraction of Co II to Fe II (Varani et al. 1990):

$\frac{M_{\mathrm{Co} \mathrm{II}}}{M_{\mathrm{Fe} \mathrm{II}}}(t)=\frac{F_{1.547}}{F_{1.533}}(t) \times \frac{1.533 A_{1.533} g_{\mathrm{Fe} \mathrm{II}} Z_{\mathrm{Co} \mathrm{II}}(t) m_{\mathrm{Co} \mathrm{II}}}{1.547 A_{1.547} g_{\mathrm{Co} \mathrm{II}} Z_{\mathrm{Fe} \mathrm{II}}(t) m_{\mathrm{Fe} \mathrm{II}}} \times e^{\frac{k_{\mathrm{B}} 2040 \mathrm{~K}}{k_{\mathrm{B}} T(t)}}$,

where $T$ is the temperature of the gas in degrees Kelvin reflecting the difference in the upper energy levels of the two transitions, $A$ are the transition probabilities between the $\mathrm{a}^{4} \mathrm{~F}_{9 / 2}-\mathrm{a}^{4} \mathrm{D}_{5 / 2} \mathrm{Fe}$ II and $a^{5} \mathrm{~F}_{5}-\mathrm{b}^{3} \mathrm{~F}_{4}$ Co II levels, $g$ are the statistical weights of the upper levels, $m$ are the atomic masses and

$Z(t)=\sum_{i}\left(g_{i} \exp \left[-E_{i} /\left(k_{\mathrm{B}} T(t)\right)\right]\right)$

are the atomic partition functions. The flux ratio, partition functions, temperature and the inferred mass ratio are functions of time after explosion. For the atomic data used in this work Eq. (1) reduces to

$\frac{M_{\mathrm{Co} \mathrm{II}}}{M_{\mathrm{Fe} \text { II }}}(t)=0.0613 \times \frac{F_{1.547}}{F_{1.533}}(t) \times \frac{Z_{\mathrm{Co} \mathrm{II}}}{Z_{\mathrm{Fe} \text { II }}}(t) \times e^{\frac{k_{\mathrm{B}} 2040 \mathrm{~K}}{k_{\mathrm{B}} T(t)}}$.

As the SN 1998bu 269 d spectrum covers only the NIR we cannot determine the temperature and electron density from the ratio of optical to NIR Fe II lines. To determine the line widths and velocity offsets we fit the spectrum for a fixed temperature $T=7200 \mathrm{~K}$ and electron density $\log \left(\mathrm{n}_{\mathrm{e}} / \mathrm{cm}^{-3}\right)=6.2$. These numbers lie between the fit results of SN $2013 \mathrm{ct}$ at $247 \mathrm{~d}$ and SN2013cs at $321 \mathrm{~d}$. The mass ratio is then computed for $T=(7200 \pm 1500) \mathrm{K}$ and $\log \left(\mathrm{n}_{\mathrm{e}} / \mathrm{cm}^{-3}\right)=(6.20 \pm 0.15)$. The uncertainties of the density and temperature are reflected in an increased uncertainty of the derived mass ratio $M_{\mathrm{Co} \text { II }} / M_{\mathrm{Fe} \mathrm{II}}$ for this spectrum.

The spectrum of SN1998bu at $364 \mathrm{~d}$ does not cover the $1.019 \mu \mathrm{m}$ Co II line, which affects the uncertainty of the inferred mass ratio significantly as the Co II $1.547 \mu \mathrm{m}$ line is rather weak compared to the Fe II $1.533 \mu \mathrm{m}$ line. Good fits can be achieved for a wide range of cobalt masses in this case.

The spectra of SN 2014J only cover the NIR wavelengths down to $8500 \AA$. We therefore cannot use the $7200 \AA[\mathrm{Fe} \mathrm{II}]+$ [Ni II] complex to determine the density and temperature in the ejecta. We find that the $8600 \AA$ feature is well reproduced (best at late epochs $>350 \mathrm{~d}$ ) by our emission model for other $\mathrm{SNe}$ in our sample with both optical and NIR wavelength coverage. We estimate the temperature and density of our SN 2014J spectra by fitting the $8600 \AA$ A feature.

In Table 4, we present the $68 \%$ credibility interval for the electron density, temperature, Co II / Fe II mass ratio, widths and shifts of [Fe II], [Co II] and [Co III]. The evolution of the mass ratio with the time since the supernova explosion is shown in Fig. 3. The error bars in the plot reflect mainly the uncertainty of the degenerate density and temperature, and, for very late spectra (>400 d), the Doppler width of singly ionised cobalt. Superimposed on the data points is the change of the mass ratio of cobalt to iron as expected from the radioactive decay of ${ }^{56} \mathrm{Ni}$ (black curve). The line is not a fit to the data but rather the evolution of the mass ratio assuming the established decay half-lives of 6.08 and 77.27 days for ${ }^{56} \mathrm{Ni}$ and ${ }^{56} \mathrm{Co}$ respectively:

${ }_{28}^{56} \mathrm{Ni} \stackrel{T_{1 / 2}=6.08 \mathrm{~d}}{\longrightarrow}{ }_{27}^{56} \mathrm{Co} \stackrel{T_{1 / 2}=77.27 \mathrm{~d}}{\longrightarrow}{ }_{26}^{56} \mathrm{Fe}$.

\subsection{Shifts and widths of singly and doubly ionised material}

We can use the velocity shifts of [Fe II], [Co II], and [Co III] to perform a similar study to Maguire et al. (2018). We present our results in Figs. 4 and 5. Different velocity shifts between stable material ([Ni $\mathrm{II}]$ ), singly ionised decay products ([Fe II], [Co II]) and doubly ionised decay products ([Fe III], [Co III]) would suggest their production in spatially separated regions of the ejecta (Maeda et al. 2010). As we fit the forbidden Co II lines at 1.019 and $1.547 \mu \mathrm{m}$ we can directly compare velocity offsets 


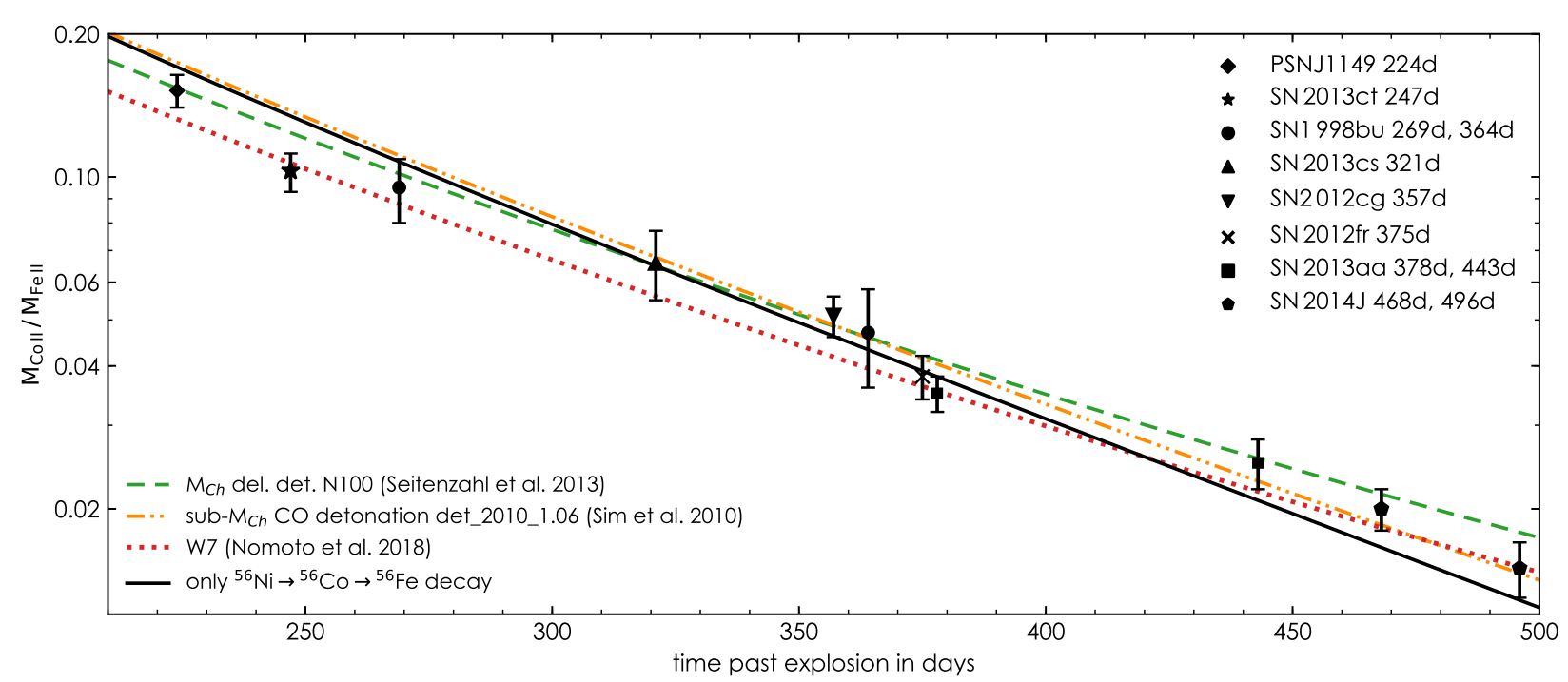

Fig. 3. Evolution of the inferred $M_{\mathrm{Co} \mathrm{II}} / M_{\mathrm{Fe} \mathrm{II}}$ ratio with time. We assumed a rise time of 18 days (Ganeshalingam et al. 2011). The error bars reflect the $68 \%$ posterior interval of the mass ratio. The coloured lines show the expected mass ratio $M_{\mathrm{Co}} / M_{\mathrm{Fe}}$ of the $\mathrm{M}_{C h}$ delayed-detonation model "N100" (Seitenzahl et al. 2013, green), the sub-M ${ }_{C h}$ CO detonation model "det_2010_1.06" (Sim et al. 2010, orange) and the M ${ }_{C h}$ "W7 $Z_{\odot}$ " model (Nomoto \& Leung 2018, red). The black line is not a fit to the data and represents the $M_{\mathrm{Co}} / M_{\mathrm{Fe}}$ ratio assuming only radioactive decay from ${ }^{56} \mathrm{Ni}$ to ${ }^{56} \mathrm{Co}$ to ${ }^{56} \mathrm{Fe}$. The same ionisation fractions of Co II and Fe II allow us to generalise $M_{\mathrm{Co} \text { II }} / M_{\mathrm{Fe} \text { II }}$ to $M_{\mathrm{Co}} / M_{\mathrm{Fe}}$ and compare the two ratios (see Sect. 5).

Table 4. 68\% posterior probability intervals of the fit parameters.

\begin{tabular}{|c|c|c|c|c|c|c|c|c|c|c|}
\hline SN & $\begin{array}{l}\mathrm{Age}^{a} \\
\text { (days) }\end{array}$ & $\begin{array}{c}\text { Mass ratio } \\
M_{\mathrm{Co} \text { II }} / M_{\mathrm{Fe} \mathrm{II}}\end{array}$ & $\log \left[\mathrm{n}_{e} / \mathrm{cm}^{-3}\right]$ & $\begin{array}{c}T \\
\left(10^{3} \mathrm{~K}\right)\end{array}$ & $\begin{array}{l}\text { Width [Fe II }]^{b} \\
\left(10^{3} \mathrm{~km} \mathrm{~s}^{-1}\right)\end{array}$ & $\begin{array}{l}\text { Shift }[\mathrm{Fe} \mathrm{II}]^{c} \\
\left(10^{3} \mathrm{~km} \mathrm{~s}^{-1}\right)\end{array}$ & $\begin{array}{c}\text { Width [Co II }{ }^{b} \\
\left(10^{3} \mathrm{~km} \mathrm{~s}^{-1}\right)\end{array}$ & $\begin{array}{l}\text { Shift [Co II }]^{c} \\
\left(10^{3} \mathrm{~km} \mathrm{~s}^{-1}\right)\end{array}$ & $\begin{array}{c}\text { Width [Co III }]^{b} \\
\left(10^{3} \mathrm{~km} \mathrm{~s}^{-1}\right)\end{array}$ & $\begin{array}{r}\text { Shift [Co III] }{ }^{6} \\
\left(10^{3} \mathrm{~km} \mathrm{~s}^{-1}\right)\end{array}$ \\
\hline PSN J1149 & 224 & $0.152_{-0.012}^{+0.012}$ & $6.39_{-0.08}^{+0.10}$ & $8.5_{-1.3}^{+2.3}$ & $7.88_{-0.55}^{+0.65}$ & $-1.74_{-0.26}^{+0.26}$ & $7.21_{-0.77}^{+0.99}$ & $-2.10_{-0.37}^{+0.36}$ & $8.63_{-0.28}^{+0.29}$ & $-0.71_{-0.16}^{+0.17}$ \\
\hline SN 2013ct & 247 & $0.103_{-0.009}^{+0.010}$ & $6.42_{-0.15}^{+0.28}$ & $7.6_{-1.6}^{+1.6}$ & $8.19_{-0.47}^{+0.053}$ & $0.28_{-0.22}^{+0.20}$ & $7.78_{-0.80}^{+0.76}$ & $0.78_{-0.39}^{+0.43}$ & $9.31_{-0.32}^{+0.34}$ & $-0.14_{-0.18}^{+0.18}$ \\
\hline SN 1998bu & 269 & $0.095_{-0.014}^{+0.015}$ & $6.20_{-0.15}^{+0.15}$ & $7.2_{-1.5}^{+1.5}$ & $6.51_{-0.44}^{+0.44}$ & $-0.94_{-0.29}^{+0.32}$ & $7.34_{-0.54}^{+0.54}$ & $-1.51_{-0.36}^{+0.36}$ & - & - \\
\hline SN 2013cs & 321 & $0.066_{-0.011}^{+0.011}$ & $6.05_{-0.10}^{+0.10}$ & $6.9_{-0.9}^{+1.0}$ & $7.66_{-0.39}^{+0.43}$ & $1.18_{-0.19}^{+0.18}$ & $7.67_{-0.85}^{+0.78}$ & $0.90_{-0.40}^{+0.37}$ & $10.42_{-0.82}^{+0.93}$ & $0.88_{-0.37}^{+0.40}$ \\
\hline SN 2012cg & 357 & $0.051_{-0.005}^{+0.005}$ & $5.81_{-0.10}^{+0.12}$ & $6.5_{-0.9}^{+0.9}$ & $7.68_{-0.22}^{+0.24}$ & $-1.48_{-0.10}^{+0.10}$ & $7.36_{-0.91}^{+0.89}$ & $-0.84_{-0.39}^{+0.35}$ & $12.30_{-1.21}^{+1.38}$ & $0.60_{-0.58}^{+0.57}$ \\
\hline SN 1998bu & 364 & $0.047_{-0.011}^{+0.011}$ & $5.63_{-0.12}^{+0.19}$ & $5.5_{-0.8}^{+0.7}$ & $9.08_{-0.40}^{+0.44}$ & $-1.08_{-0.16}^{+0.16}$ & $8.52_{-0.48}^{+0.54}$ & $-1.31_{-0.88}^{+0.97}$ & $12.41_{-1.21}^{+1.37}$ & $-1.03_{-0.59}^{+0.68}$ \\
\hline SN 2012fr & 375 & $0.038_{-0.004}^{+0.004}$ & $5.77_{-0.19}^{+0.16}$ & $5.7_{-0.8}^{+1.5}$ & $7.17_{-0.18}^{+0.19}$ & $1.42_{-0.10}^{+0.10}$ & $6.97_{-0.63}^{+0.97}$ & $0.65_{-0.30}^{+0.28}$ & $10.16_{-0.95}^{+1.12}$ & $0.58_{-0.59}^{+0.55}$ \\
\hline SN 2013aa & 378 & $0.035_{-0.003}^{+0.003}$ & $5.67_{-0.11}^{+0.14}$ & $6.6_{-1.1}^{+1.1}$ & $7.32_{-0.22}^{+0.24}$ & $-1.35_{-0.09}^{+0.10}$ & $7.51_{-0.65}^{+0.71}$ & $-0.91_{-0.29}^{+028}$ & $10.29_{-1.08}^{+1.57}$ & $-0.13_{-0.55}^{+0.53}$ \\
\hline SN 2013aa & 443 & $0.025_{-0.003}^{+0.003}$ & $5.44_{-0.16}^{+0.28}$ & $5.0_{-0.9}^{+0.7}$ & $7.87_{-0.17}^{+0.18}$ & $-1.18_{-0.07}^{+0.07}$ & $8.19_{-0.48}^{+0.46}$ & $-1.06_{-0.22}^{+0.22}$ & $12.07_{-1.88}^{+1.86}$ & $0.15_{-0.86}^{+0.92}$ \\
\hline SN 2014J & 468 & $0.020_{-0.002}^{+0.002}$ & $5.24_{-0.16}^{+0.28}$ & $4.3_{-0.9}^{+0.7}$ & $8.59_{-0.14}^{+0.14}$ & $0.45_{-0.06}^{+0.06}$ & $9.13_{-0.32}^{+0.31}$ & $0.36_{-0.13}^{+0.13}$ & - & - \\
\hline SN 2014J & 496 & $0.015_{-0.002}^{+0.002}$ & $5.15_{-0.15}^{+0.10}$ & $3.8_{-0.3}^{+0.4}$ & $9.15_{-0.14}^{+0.14}$ & $0.67_{-0.07}^{-0.07}$ & $8.75_{-0.66}^{+0.32}$ & $0.03_{-0.29}^{+0.15}$ & - & - \\
\hline$\overline{\text { Mean }+ \text { Std }^{d}}$ & & & & & $7.9 \pm 0.8$ & $1.1 \pm 0.5$ & $7.9 \pm 1.0$ & $0.9 \pm 0.7$ & $10.7 \pm 1.8$ & $0.5 \pm 0.6$ \\
\hline
\end{tabular}

Notes. For SN 1998 bu at 269 days the temperature and density are not fitted (see text for details). ${ }^{(a)}$ Days after the explosion, assuming a rise time of $\sim 18$ days (Ganeshalingam et al. 2011). ${ }^{(b)}$ Doppler full width at half maximum. ${ }^{(c)}$ Shift velocity. ${ }^{(d)}$ Mean and standard deviation over all fit samples. For shift velocities we calculated the mean and standard deviation of the absolute values.

and widths of singly and doubly ionised cobalt. For Fe III and Fe II this cannot be done as the $4700 \AA$ Fe III-dominated feature exhibits a shift of its central wavelength with time.

We find that the absolute velocity shifts of [Fe II], $(1.1 \pm$ $0.5) \times 10^{3} \mathrm{~km} \mathrm{~s}^{-1}$, are very similar to the absolute velocity shifts of [Co II $](0.9 \pm 0.7) \times 10^{3} \mathrm{~km} \mathrm{~s}^{-1}$. Deviations of the velocity shift between the two ions are less than $500 \mathrm{~km} \mathrm{~s}^{-1}$ for most spectra and likely the result of noise near the $1.019 \mu \mathrm{m}$ [Co II] feature. Velocity shifts of singly ionised ions can be either positive or negative for the observed sample.

Lines of Fe II and Co II exhibit comparable widths. Line widths of [Co II] usually have a higher uncertainty than line widths of $[\mathrm{Fe} \mathrm{II}]$ as there is only one unblended Co II feature in our spectra, but multiple features of [Fe II] can be found at $7200,8600,12600$ and $16400 \AA$. We find widths of $(7.9 \pm 1.0) \times 10^{3} \mathrm{~km} \mathrm{~s}^{-1}$ for $[\mathrm{Co} \mathrm{II}]$ and $(7.9 \pm 0.8) \times 10^{3} \mathrm{~km} \mathrm{~s}^{-1}$ for $[\mathrm{Fe} \mathrm{II}]$.

We find that [Co III] shows lower velocity offsets from its rest wavelength than singly ionised iron and cobalt. While there are spectra in which the [Co III] velocity shift is similar to [Fe II] and [Co II] (SN 2013cs, SN 1998bu), for the majority of spectra in our sample we get different velocity shifts between [Co III] and [Co II]. We find $(0.5 \pm 0.6) \times 10^{3} \mathrm{~km} \mathrm{~s}^{-1}$ for the mean absolute [Co III] velocity shift, compared to $(0.9 \pm 0.7) \times 10^{3} \mathrm{~km} \mathrm{~s}^{-1}$ for [Co II]. As noted in Maguire et al. (2018), shifts of [Co III] seem to be consistent with zero offset from the rest wavelength.

On average, [Co III] lines also appear to be broader than [Co II] lines by about $3000 \mathrm{~km} \mathrm{~s}^{-1}\left((10.7 \pm 1.8) \times 10^{3} \mathrm{~km} \mathrm{~s}^{-1}\right.$ 


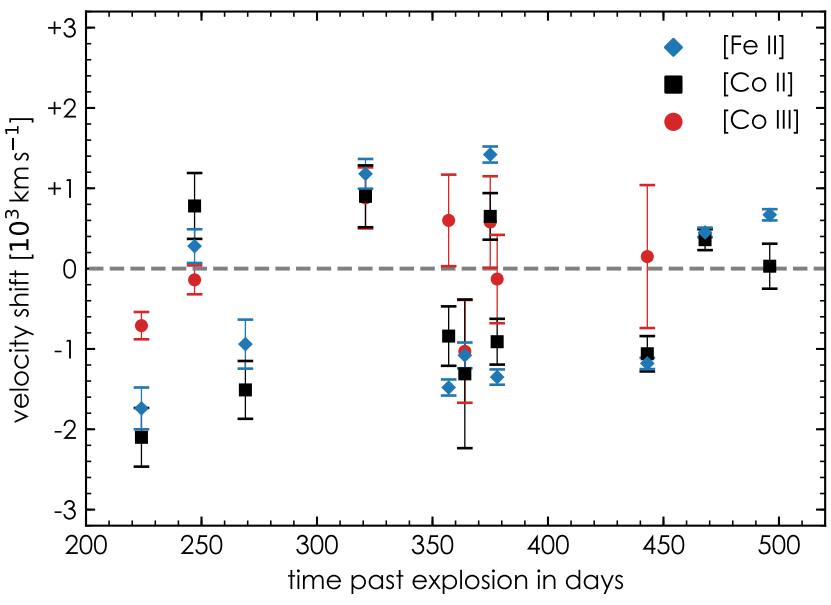

Fig. 4. Velocity shifts of [Fe II], [Co II], and [Co III] as a function of time after explosion.

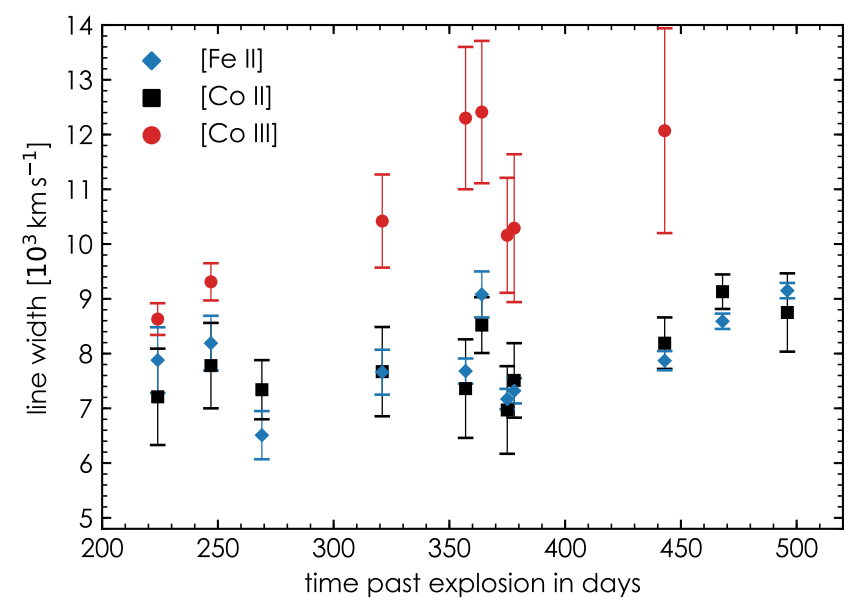

Fig. 5. Line widths of [Fe II], [Co II], and [Co III] as a function of time after explosion.

for [Co III] compared to $(7.9 \pm 1.0) \times 10^{3} \mathrm{~km} \mathrm{~s}^{-1}$ for [Co II] $)$. We note that supernovae which exhibit a strong continuum in the 5900 A region (SN 2012cg, SN 1998bu at 364 d, SN 2013aa at $443 \mathrm{~d}$ ) tend to have higher line widths than supernovae without continuum in this region. In these cases, the line widths appear to be strongly affected by the manual continuum subtraction. [Co III] widths determined in this work are compatible within their uncertainties with results from Maguire et al. (2018). [Co III] lines in our fits are redshifted by $\sim 500 \mathrm{~km} \mathrm{~s}^{-1} \mathrm{com}-$ pared to Maguire et al. (2018). This systematic shift is mostly due to the presence of [Fe II] lines from the $a^{4} \mathrm{~F}-\mathrm{a}^{2} \mathrm{D}$ multiplet $(5335,5377,5528 \AA)$ on the blue side of the $5900 \AA$ feature which are not included in the work of Maguire et al. (2018). We note that the choice of the continuum differs between this work and Maguire et al. (2018).

\section{Comparison of $M_{\mathrm{CoII}} / M_{\mathrm{Fe} \text { II }}$ with explosion model yields}

Besides ${ }^{56} \mathrm{Ni}$, explosion models predict the presence of the isotopes ${ }^{54,56} \mathrm{Fe},{ }^{57} \mathrm{Ni}$ and ${ }^{58} \mathrm{Ni}$. If the explosive burning in the white dwarf occurs at high densities (e.g. $\mathrm{M}_{C h}$ models) stable ${ }^{54,56} \mathrm{Fe}$ and ${ }^{58} \mathrm{Ni}$ and rapidly decaying ${ }^{57} \mathrm{Ni}$ can be produced directly.
Table 5. Models and their included isotopes produced in the explosion.

\begin{tabular}{cccc}
\hline \hline Model & ${ }^{56} \mathrm{Ni}$ & ${ }^{54,56} \mathrm{Fe}$ & ${ }^{57} \mathrm{Ni}$ \\
\hline$M_{0}$ & $\checkmark$ & $x$ & $x$ \\
$M_{1}$ & $\checkmark$ & $\checkmark$ & $x$ \\
$M_{2}$ & $\checkmark$ & $x$ & $\checkmark$ \\
$M_{3}$ & $\checkmark$ & $\checkmark$ & $\checkmark$ \\
\hline
\end{tabular}

Sub- $\mathrm{M}_{C h}$ models lack a high enough central density to produce neutron-rich material. However, if the progenitor has a high metallicity (several times solar) there is an excess of neutrons, resulting in the synthesis of ${ }^{54,56} \mathrm{Fe},{ }^{57} \mathrm{Ni}$, and stable ${ }^{58} \mathrm{Ni}$ with masses comparable to those of $\mathbf{M}_{C h}$ explosions (Sim et al. 2010; Ruiter et al. 2013).

In Fig. 3 the prediction of the $M_{\mathrm{Co}} / M_{\mathrm{Fe}}$ ratio is shown in the case of pure ${ }^{56} \mathrm{Ni}$ to ${ }^{56} \mathrm{Co}$ to ${ }^{56} \mathrm{Fe}$ decay and for nucleosynthetic yields of the $\mathrm{M}_{C h}$ deflagration model "W7 $Z_{\odot}$ " (Nomoto \& Leung 2018), the $\mathrm{M}_{C h}$ delayed-detonation model "N100" (Seitenzahl et al. 2013), and the sub-M $\mathrm{M}_{C h} \mathrm{CO}$ detonation model "det_2010_1.06" (Sim et al. 2010). The "W7 $Z_{\odot}$ " model produces $2.8 \%{ }^{57} \mathrm{Ni}$ and $35 \%{ }^{54,56} \mathrm{Fe}$, the $\mathrm{M}_{C h}$ delayed detonation model "N100" produces $3.1 \%{ }^{57} \mathrm{Ni}$ and $19.4 \%{ }^{54,56} \mathrm{Fe}$, and the sub-M $\mathrm{M}_{C h} \mathrm{CO}$ detonation model 'det_2010_1.06' produces $0.6 \%{ }^{57} \mathrm{Ni}$ and no stable iron, each given in fractions of the ${ }^{56} \mathrm{Ni}$ mass. As mentioned before, a high metallicity progenitor like in the "det_2010_1.06_0.075Ne" model (Sim et al. 2010) produces similar masses of ${ }^{54,56} \mathrm{Fe}$ and ${ }^{57} \mathrm{Ni}$ as the "N100" model.

Stable iron produced in the explosion has the strongest effect on $M_{\mathrm{Co}} / M_{\mathrm{Fe}}$ while not all of the ${ }^{56} \mathrm{Co}$ has yet decayed to ${ }^{56} \mathrm{Fe}$ $(<300 \mathrm{~d}) .{ }^{57} \mathrm{Ni}$ decays within a few days to ${ }^{57} \mathrm{Co}$, which has a much longer half-life than ${ }^{56} \mathrm{Co}$ :

${ }_{28}^{57} \mathrm{Ni} \stackrel{T_{1 / 2}=1.48 \mathrm{~d}}{\longrightarrow}{ }_{27}^{57} \mathrm{Co} \stackrel{T_{1 / 2}=271.74 \mathrm{~d}}{\longrightarrow}{ }_{26}^{57} \mathrm{Fe}$.

Due to the longer half-life it can still be found late in the nebular phase $(>400 \mathrm{~d})$ when almost no ${ }^{56}$ Co remains.

Co II and Fe II have similar ionisation potentials and appear to be co-located within the ejecta (see Sect. 4). We therefore assume that the relative ionisation fraction between Co II and Fe II does not change with time, allowing us to generalise $M_{\mathrm{Co} I \mathrm{II}} / M_{\mathrm{Fe} \text { II }}$ to $M_{\mathrm{Co}} / M_{\mathrm{Fe}}$. This ratio measures the total mass of cobalt to iron at a given time, with possible contributions from stable ${ }^{54,56} \mathrm{Fe}$ and decay products according to Eqs. (4) and (5). We compare the predicted $\mathrm{M}_{\mathrm{Co}} / \mathrm{M}_{\mathrm{Fe}}$ ratio of different models with the results from our fits by determining their likelihood based on their Bayes factors. The Bayes factor quantifies the evidence of data $D$ for model $M_{a}$ compared to model $M_{b}$. In our analysis we consider the nested models $M_{0}$ to $M_{3}$ (see Table 5). The possible parameters of these models are initial abundances of the isotopes ${ }^{56} \mathrm{Ni},{ }^{54,56} \mathrm{Fe}$, and ${ }^{57} \mathrm{Ni}$.

For nested models, the Bayes factor can be computed analytically if the priors are separable and the more complicated model $M_{b}$ becomes the simpler model $M_{a}$ for $\theta=\theta_{0}$ :

$B_{a b}=\frac{P\left(\theta=\theta_{0} \mid D, M_{b}\right)}{P\left(\theta=\theta_{0} \mid M_{b}\right)}$.

We use the mass ratio results from our individual spectral fits to compute the Bayes factor between models $M_{0}$ and $M_{3}$. Prior odds are set to one as we do not have an initial preference for any model. For ${ }^{54,56} \mathrm{Fe}$ and ${ }^{57} \mathrm{Ni}$ we adopt flat priors $0 \leq$ $M_{54,56 \mathrm{Fe}} / M_{56 \mathrm{Ni}} \leq 0.5$ and $0 \leq M_{57} \mathrm{Ni} / M_{56 \mathrm{Ni}} \leq 0.05$. The covariances and posterior densities of the models are shown in Fig. 6 


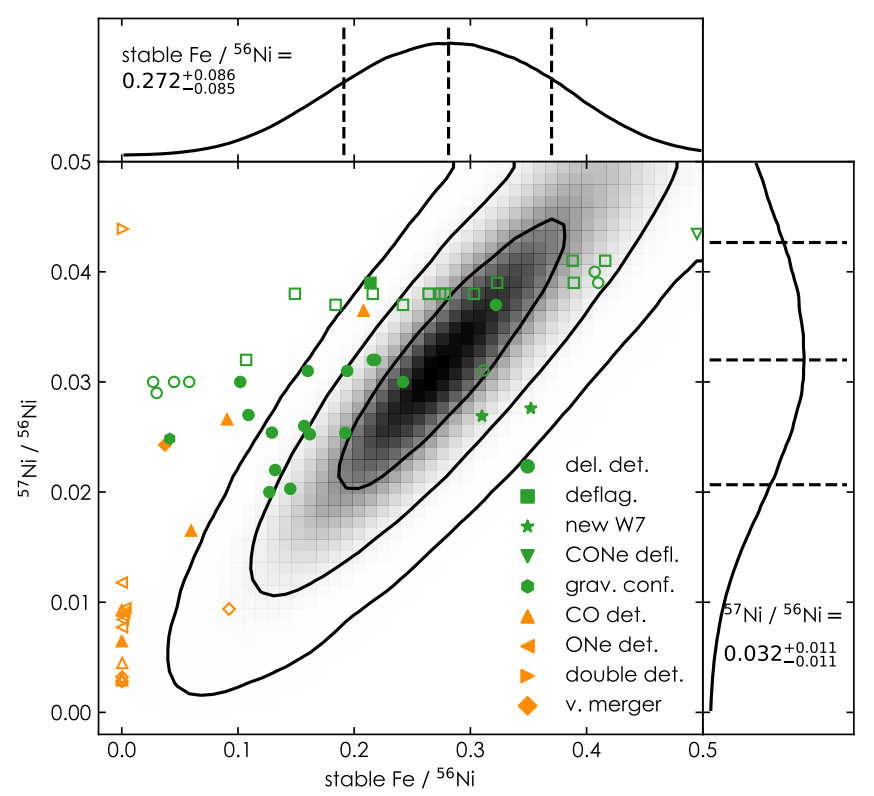

Fig. 6. Covariances and normalized posterior densities of model $M_{3}$. Theoretical predictions of the initial ${ }^{54,56} \mathrm{Fe}$ and ${ }^{57} \mathrm{Ni}$ fraction of various explosion models are indicated by green (Near-Ch-mass) and orange (sub-Ch-mass) symbols. Theoretical predictions with too little $\left(<0.4 M_{\odot}\right)$ or too much $\left(>0.8 M_{\odot}\right){ }^{56} \mathrm{Ni}$ compared to Branch-normal $\mathrm{SNe}$ Ia are marked as empty symbols. Black lines show the 1,2 , and $3 \sigma$ credibility regions. Numbers next to the $1 \mathrm{D}$ histograms indicate the $68 \%$ credibility range of the individual parameters. Models: Del. Det. (Seitenzahl et al. 2013; Ohlmann et al. 2014; Nomoto \& Leung 2018), Deflag. (Fink et al. 2014), new W7 (Nomoto \& Leung 2018), CONe Defl. (Kromer et al. 2015), Grav. Conf. (Seitenzahl et al. 2016), CO Det. (Sim et al. 2010; Nomoto \& Leung 2018), ONe Det. (Marquardt et al. 2015), Double Det. (Sim et al. 2012), V. Merger (Pakmor et al. 2010, 2012; Kromer et al. 2013, 2016).

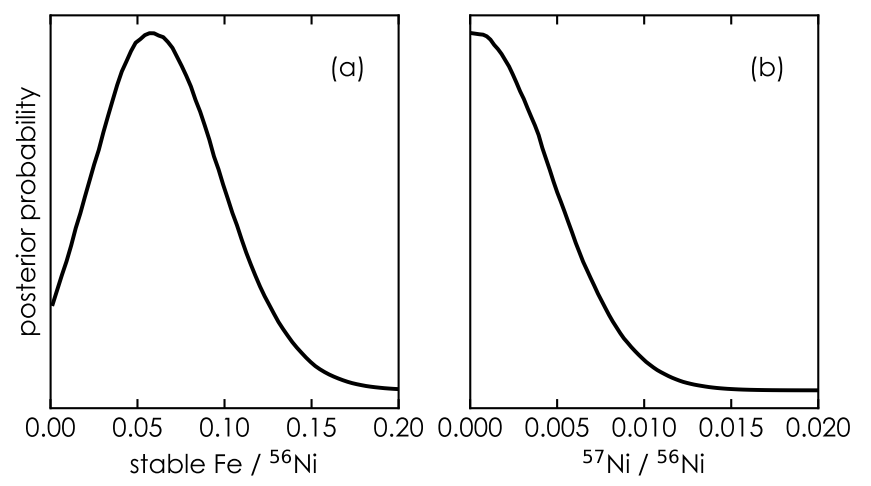

Fig. 7. Panel a: normalized posterior density of model $M_{1}$ with only stable iron ${ }^{54,56} \mathrm{Fe}$ and radioactive ${ }^{56} \mathrm{Ni}$. Panel $b$ : normalized posterior density of model $M_{2}$ with only radioactive ${ }^{57} \mathrm{Ni}$ and ${ }^{56} \mathrm{Ni}$.

for $M_{3}$ and in Fig. 7 for $M_{1}$ and $M_{2}$. Due to the introduction of additional degrees of freedom all models fit the data reasonably well.

To determine the best fitting model given the data we compute the Bayes factors between the models as shown in Table 6 . The $\mathrm{M}_{\mathrm{Co}} / \mathrm{M}_{\mathrm{Fe}}$ ratio of both $\mathrm{M}_{C h}$ and sub- $\mathrm{M}_{C h}$ explosion models follows closely the mass ratio from pure ${ }^{56} \mathrm{Ni}$ decay between 250 and 350 days after the explosion. Data points between these epochs cannot distinguish between either model.

Most $\mathrm{M}_{C h}$ explosion models that predict between 0.4 and $0.8 M_{\odot}$ of ${ }^{56} \mathrm{Ni}$ (filled symbols in Fig. 6) lie in the
Table 6. Bayes factors and their interpretation between the nested models and relative probabilities of the models.

\begin{tabular}{|c|c|c|c|c|c|}
\hline Bayes F. ${ }^{a}$ & $\ln (\mathrm{B})$ & Pref. ${ }^{b}$ & Interpr. $^{c}$ & Model & Rel. Prob. ${ }^{d}$ \\
\hline$B_{01}$ & +0.6 & $M_{0}$ & Weak & & 0.022 \\
\hline$B_{02}$ & +2.1 & $M_{0}$ & Positive & $\begin{array}{l}M_{0} \\
M_{1}\end{array}$ & $\begin{array}{l}0.022 \\
0.013\end{array}$ \\
\hline$B_{03}$ & -3.8 & $M_{3}$ & Strong & $\begin{array}{l}M_{1} \\
M_{2}\end{array}$ & $\begin{array}{l}0.013 \\
0.002\end{array}$ \\
\hline$B_{13}$ & -4.1 & $M_{3}$ & Strong & $M_{2}$ & 0.063 \\
\hline$B_{23}$ & -5.8 & $M_{3}$ & Very strong & & \\
\hline
\end{tabular}

Notes. ${ }^{(a)}$ Bayes Factor $B_{a b}$ between models $M_{a}$ and $M_{b} .{ }^{(b)}$ Preferred model. ${ }^{(c)}$ Interpretation according to Kass \& Raftery (1995). ${ }^{\left({ }^{(d)}\right.}$ Relative probability $P\left(D \mid M_{a}\right) / \sum P\left(D \mid M_{i}\right)$.

$3 \sigma$ error ellipse of model $M_{3}$. The error ellipse also contains the aforementioned high-metallicity $\mathrm{CO}$ detonation model "det_2010_1.06_0.075Ne" (Sim et al. 2010). Models, that yield less than $0.4 M_{\odot}$ or more than $0.8 M_{\odot}$ of ${ }^{56} \mathrm{Ni}$ are marked with empty symbols. The models with only ${ }^{57} \mathrm{Ni}$ or only ${ }^{54,56} \mathrm{Fe}$ in addition to the ${ }^{56} \mathrm{Ni}$ are not allowed as the gain in fit quality is small compared to the added parameter space (see Fig. 7). A comparison between models ${ }^{56} \mathrm{Ni}$ only $\left(M_{0}\right)$ and our more complex $M_{3}$ model favours the complex model.

\section{Discussion}

\subsection{Emission line widths and velocity shifts}

Maguire et al. (2018) found that the velocity shifts and line widths of [Fe II] and [Ni II] of SNe in the sample are compatible within their uncertainties. We measure an average [Fe II] velocity shift of $(1.1 \pm 0.5) \times 10^{3} \mathrm{~km} \mathrm{~s}^{-1}$, across the sample, compared to $(0.9 \pm 0.7) \times 10^{3} \mathrm{~km} \mathrm{~s}^{-1}$ for [Co II]. Lines of [Fe II] and [Co II] are either both redshifted or both blueshifted. The average line widths are $(7.9 \pm 1.0) \times 10^{3} \mathrm{~km} \mathrm{~s}^{-1}$ in the case of [Co II $]$ and $(7.9 \pm 0.8) \times 10^{3} \mathrm{~km} \mathrm{~s}^{-1}$ for $[\mathrm{Fe} \mathrm{II}]$. This indicates that the emission from all singly ionised iron-group ions originates from the same spatial region in the ejecta. The emitting region of singly ionised material appears to be located off-centre and is smaller than the emitting region of doubly ionised cobalt by about $3000 \mathrm{~km} \mathrm{~s}^{-1}$. We do not find significant velocity shift and line width offsets between [Fe II] emission in the optical and NIR. We confirm the results of Maguire et al. (2018) that the absolute velocity shift of [Co III] is lower than that of the singly ionised species and for most spectra in our sample compatible with no offset from the rest wavelength.

Nucleosynthetic calculations for delayed detonations including multiple ignition points by Seitenzahl et al. (2013) show the stable iron group ${ }^{54} \mathrm{Fe},{ }^{56} \mathrm{Fe}$, and ${ }^{58} \mathrm{Ni}$ to be distributed within the supernova in close association with ${ }^{56} \mathrm{Ni}$. At the epochs of our observations ( $\sim 200-500$ days), the $\gamma$-rays still deposit energy into the ejecta and any stable iron, whether in the core or distributed within the ejecta, should be heated and contribute to the emission. If stable and radioactive materials are co-located in the same region, the stable material can also be heated by positrons. In fact, evidence for heating of stable elements is found in the late-time optical spectra where the line of [Ni II] is evident at $7378 \AA$ (e.g. Maeda et al. 2010; Maguire et al. 2018). No significant amount of ${ }^{56} \mathrm{Ni}$ can be expected to be present in the ejecta 100 days after the explosion.

It is possible to hide a large amount of material if it is not heated and therefore not emitting. At epochs later than 350 days, when the mean free path for the $\gamma$-rays from the decay of ${ }^{56} \mathrm{Co}$ is 
larger than the radius of the supernova and the bulk of the $\gamma$-rays do not deposit their energy in the ejecta, the energy deposition within the ejecta is almost exclusively via the $\beta^{+}$channel (e.g. Axelrod 1980; Woosley et al. 1989, 2007; Leibundgut \& Pinto 1992). It is assumed, due to trapping by magnetic fields, that the positron energy deposition is local to the emission region. It is, therefore, possible to have pockets of cold gas. In such a case those regions would not contribute to the spectra. Unlike the observations of SN 2003hv (Motohara et al. 2006; Maeda et al. 2010; Mazzali et al. 2011), as discussed in Maguire et al. (2018) our spectra do not exhibit flat-topped profiles of emission features and there is no evidence for an ashes bubble in the centre of, or off-centre, in the ejecta. A more sophisticated analysis of the line profiles of these species in the context of energy deposition scenarios and distributions can be found in Diamond et al. (2018).

\subsection{The origin of iron and cobalt}

Doubly ionised cobalt and iron lines have been used in the past by other authors (see Kuchner et al. 1994) to derive that the mass ratio $\mathrm{M}_{\mathrm{Co}} / \mathrm{M}_{\mathrm{Fe}}$ in $\mathrm{SNe} \mathrm{Ia}$ is governed by the radioactive decay of ${ }^{56} \mathrm{Co}$ to ${ }^{56} \mathrm{Fe}$. That work relied on iron and cobalt lines below $5500 \AA$ A. Singly ionised lines above $7000 \AA$ A become optically thin after about 150 days.

Our work determines that the singly ionised lines observed in the spectra arise from the daughter products of the radioactive ${ }^{56} \mathrm{Ni}$. Even though we can only constrain the temperature weakly, it is evident that the mass ratio evolution is not a temperature effect. While it is plausible to assume variations in the temperature of the ejecta from one SN to another, these are unlikely to be extreme as the heating and cooling are fundamentally dictated by the same elements (the daughter products of the decay of ${ }^{56} \mathrm{Ni}$ ). Furthermore, the production of ${ }^{56} \mathrm{Ni}$ is tightly linked to the density and temperature of the progenitor. Simply put, the inner ejecta of normal SNe Ia should be very similar in terms of their excitation within the boundaries of this analysis. This is borne out by the very strong similarity of the emission line spectra obtained during the first year after the explosion (Mazzali et al. 1998).

By fitting the spectra with forbidden emission lines of our set of NLTE atoms we find a decline of the temperature in the ejecta from $\sim 8000 \mathrm{~K}$ at 200 days to $\sim 4000 \mathrm{~K}$ at 500 days. Such a temperature evolution is consistent with predictions from simulations (Fransson \& Jerkstrand 2015). We also find that the density of the emitting material decreases from a few times $10^{6} \mathrm{~cm}^{-3}$ to about $10^{5} \mathrm{~cm}^{-3}$ between the youngest and oldest spectrum in our sample. These densities are consistent with a simple distribution of roughly half a solar mass of singly and doubly ionised iron group material in a volume expanding at $\sim 8000 \mathrm{~km} \mathrm{~s}^{-1}$ for the age of the SN.

We ruled out models containing only stable iron or only ${ }^{57} \mathrm{Ni}$ in addition to ${ }^{56} \mathrm{Ni}$. Theoretical predictions of the cobalt to iron mass ratio from $\mathbf{M}_{C h}$ and sub- $\mathbf{M}_{C h}$ explosion simulations differ only slightly from the pure ${ }^{56} \mathrm{Ni}$ decay chain. Within the Bayesian framework we find evidence $\left(B_{03}=-3.8\right)$ for a model with ${ }^{56} \mathrm{Ni}$, stable iron and ${ }^{57} \mathrm{Ni}$ produced during the explosion similar to most $\mathrm{M}_{\mathrm{Ch}}$ models, compared to a purely ${ }^{56} \mathrm{Ni}$ origin of iron.

Due to largely unknown ejecta densities and temperatures, the uncertainties of the derived cobalt to iron mass ratio are quite large. Future observations of the relative strength of the [Co II] 10.52, 14.74 and $15.46 \mu \mathrm{m}$ lines (e.g. with JWST) would also allow for a direct measurement of the density and temperature of the emitting material in the range $10^{4}-10^{6} \mathrm{~cm}^{-3}$ and $3000-10000 \mathrm{~K}$ (Storey et al. 2016). A more accurate Co II / Fe II mass ratio could be used to determine the amount of ${ }^{57} \mathrm{Ni}$ and ${ }^{54,56} \mathrm{Fe}$ for individual $\mathrm{SNe}$ rather than assuming similar progenitor scenarios for a sample of $\mathrm{SNe}$.

The presence of ${ }^{57} \mathrm{Ni}$ was also discussed for the nearby SN 2011fe (Dimitriadis et al. 2017; Kerzendorf et al. 2017; Shappee et al. 2017) and SN 2012cg (Graur et al. 2016). For these two objects photometric measurements were obtained at extremely late phases ( $>1000$ days after explosion) to construct a pseudo-bolometric light curve. Graur et al. (2016) claimed that ${ }^{57} \mathrm{Ni}$ is required to explain the late-time light curve of SN $2012 \mathrm{cg}$. Both Dimitriadis et al. (2017) and Shappee et al. (2017) argued for a detection of ${ }^{57} \mathrm{Ni}$ in SN 2011fe, albeit with very different abundances, pointing to either near- $\mathrm{M}_{C h}$ (Dimitriadis et al. 2017) or sub- $\mathrm{M}_{C h}$ explosions (Shappee et al. 2017). Kerzendorf et al. (2017) tested the effect of various isotopic abundances and physical processes on the light curve and came to the conclusion that one cannot determine the ${ }^{57} \mathrm{Ni}$ abundance from the light curve without detecting the mid-IR cooling lines.

\section{Conclusions}

We fitted late time spectra of SNe Ia with broadened emission profiles from NLTE level populations using a Bayesian sampler. We computed good fits with similar widths and velocity offsets among the singly ionised atoms, indicating a common emission region. We find that doubly ionised cobalt is located in a broader and more centred region of the ejecta. We have shown that the singly ionised iron group elements in the late-time spectra of $\mathrm{SNe}$ Ia change their flux ratios in agreement with an evolution of their mass ratios that would be governed by the radioactive decay of ${ }^{56} \mathrm{Co}$ to ${ }^{56} \mathrm{Fe}$. This result disfavours the presence of only stable iron or only the decay products of ${ }^{57} \mathrm{Ni}$ in the ejecta in addition to ${ }^{56} \mathrm{Ni}$, at least for the $\mathrm{SNe}$ observed here. For a model that produced both stable iron and ${ }^{57} \mathrm{Ni}$ in addition to ${ }^{56} \mathrm{Ni}$ during the explosion we find ${ }^{54,56} \mathrm{Fe} /{ }^{56} \mathrm{Ni}=0.272 \pm 0.086$ and ${ }^{57} \mathrm{Ni} /{ }^{56} \mathrm{Ni}=$ $0.032 \pm 0.011$ with a ratio of stable iron to ${ }^{57} \mathrm{Ni}$ of $\sim 8.5: 1$.

Acknowledgements. We would like to thank the anonymous referee for the helpful comments. We thank the staff at Gemini and Paranal observatories. This research would not be possible without their efforts in supporting queue/service mode observing. This research has made use of the NASA/IPAC Extragalactic Database (NED) which is operated by the Jet Propulsion Laboratory, California Institute of Technology, under contract with the National Aeronautics and Space Administration. This work made use of the Heidelberg Supernova Model Archive (HESMA), https://hesma.h-its.org. We would like to thank Johannes Buchner for helpful discussions and comments. A.F. acknowledges the support of an ESO Studentship. K.M. is supported by the UK STFC through an Ernest Rutherford fellowship. S.T. acknowledges support by TRR33 "The Dark Universe" of the German Research Foundation (DFG). W.E.K. acknowledges the Excellence Cluster Universe, Technische Universität München, Boltzmannstrasse 2, D-85748 Garching, Germany and acknowledges the support of an ESO Fellowship.

\section{References}

Amanullah, R., Goobar, A., Johansson, J., et al. 2014, ApJ, 788, L21 Arnett, W. D. 1982, ApJ, 253, 785

Axelrod, T. S. 1980, Ph.D. Thesis, California University, Santa Cruz, USA

Bautista, M. A., Fivet, V., Ballance, C., et al. 2015, ApJ, 808, 174

Black, C. S., Fesen, R. A., \& Parrent, J. T. 2016, MNRAS, 462, 649

Botyánszki, J., \& Kasen, D. 2017, ApJ, 845, 176

Branch, D., Dang, L. C., Hall, N., et al. 2006, PASP, 118, 560

Cappellaro, E., Patat, F., Mazzali, P. A., et al. 2001, ApJ, 549, L215

Cassidy, C. M., Ramsbottom, C. A., Scott, M. P., \& Burke, P. G. 2010, A\&A, 513, A55

Cassidy, C. M., Hibbert, A., \& Ramsbottom, C. A. 2016, A\&A, 587, A107 
Chambers, K. C., Magnier, E. A., Metcalfe, N., et al. 2016, ArXiv e-prints [arXiv: 1612.05560$]$

Childress, M. J., Hillier, D. J., Seitenzahl, I., et al. 2015, MNRAS, 454, 3816

Churazov, E., Sunyaev, R., Isern, J., et al. 2014, Nature, 512, 406

Colgate, S. A., \& McKee, C. 1969, ApJ, 157, 623

Dhawan, S., Flörs, A., Leibundgut, B., et al. 2018, A\&A, 619, A102

Diamond, T. R., Hoeflich, P., Hsiao, E. Y., et al. 2018, ApJ, 861, 119

Dimitriadis, G., Sullivan, M., Kerzendorf, W., et al. 2017, MNRAS, 468, 3798

Filippenko, A. V., Richmond, M. W., Branch, D., et al. 1992a, AJ, 104, 1543

Filippenko, A. V., Richmond, M. W., Matheson, T., et al. 1992b, ApJ, 384, L15

Fink, M., Kromer, M., Seitenzahl, I. R., et al. 2014, MNRAS, 438, 1762

Fransson, C., \& Chevalier, R. A. 1989, ApJ, 343, 323

Fransson, C., \& Jerkstrand, A. 2015, ApJ, 814, L2

Ganeshalingam, M., Li, W., \& Filippenko, A. V. 2011, MNRAS, 416, 2607

Graur, O., Zurek, D., Shara, M. M., et al. 2016, ApJ, 819, 31

Hoyle, F., \& Fowler, W. A. 1960, ApJ, 132, 565

Jha, S., Garnavich, P. M., Kirshner, R. P., et al. 1999, ApJS, 125, 73

Kass, R. E. \& Raftery, A. E. 1995, J. Am. Stat. Assoc., 90, 773

Kerzendorf, W. E., McCully, C., Taubenberger, S., et al. 2017, MNRAS, 472, 2534

Kochanek, C. S., Shappee, B. J., Stanek, K. Z., et al. 2017, PASP, 129, 104502

Kozma, C., Fransson, C., Hillebrandt, W., et al. 2005, A\&A, 437, 983

Kramida, A., Yu. Ralchenko, Reader, J., \& NIST ASD Team 2018, NIST Atomic Spectra Database (ver. 5.5.6), Available at https://physics. nist.gov/asd (Gaithersburg, MD: National Institute of Standards and Technology)

Kromer, M., Pakmor, R., Taubenberger, S., et al. 2013, ApJ, 778, L18

Kromer, M., Ohlmann, S. T., Pakmor, R., et al. 2015, MNRAS, 450, 3045

Kromer, M., Fremling, C., Pakmor, R., et al. 2016, MNRAS, 459, 4428

Kuchner, M. J., Kirshner, R. P., Pinto, P. A., \& Leibundgut, B. 1994, ApJ, 426, 89

Kulkarni, S. R. 2012, ArXiv e-prints [arXiv:1202 . 2381]

Leibundgut, B. \& Pinto, P. A. 1992, ApJ, 401, 49

Leibundgut, B. \& Sullivan, M. 2018, Space Sci. Rev., 214, A57

Leibundgut, B., Kirshner, R. P., Phillips, M. M., et al. 1993, AJ, 105, 301

Maeda, K., Taubenberger, S., Sollerman, J., et al. 2010, ApJ, 708, 1703
Maguire, K., Taubenberger, S., Sullivan, M., \& Mazzali, P. A. 2016, MNRAS, 457, 3254

Maguire, K., Sim, S. A., Shingles, L., et al. 2018, MNRAS, 477, 3567

Marquardt, K. S., Sim, S. A., Ruiter, A. J., et al. 2015, A\&A, 580, A118

Mazzali, P. A., Cappellaro, E., Danziger, I. J., Turatto, M., \& Benetti, S. 1998, ApJ, 499, L49

Mazzali, P. A., Maurer, I., Stritzinger, M., et al. 2011, MNRAS, 416, 881

Motohara, K., Maeda, K., Gerardy, C. L., et al. 2006, ApJ, 652, L101

Nomoto, K., \& Leung, S.-C. 2018, Space Sci. Rev., 214, 67

Ohlmann, S. T., Kromer, M., Fink, M., et al. 2014, A\&A, 572, A57

Pakmor, R., Kromer, M., Röpke, F. K., et al. 2010, Nature, 463, 61

Pakmor, R., Kromer, M., Taubenberger, S., et al. 2012, ApJ, 747, L10

Phillips, M. M. 1993, ApJ, 413, L105

Phillips, M. M., Wells, L. A., Suntzeff, N. B., et al. 1992, AJ, 103, 1632

Ruiter, A. J., Sim, S. A., Pakmor, R., et al. 2013, MNRAS, 429, 1425

Ruiz-Lapuente, P., Cappellaro, E., Turatto, M., et al. 1992, ApJ, 387, L33

Schlafly, E. F., \& Finkbeiner, D. P. 2011, ApJ, 737, 103

Seitenzahl, I. R., Ciaraldi-Schoolmann, F., Röpke, F. K., et al. 2013, MNRAS, 429, 1156

Seitenzahl, I. R., Kromer, M., Ohlmann, S. T., et al. 2016, A\&A, 592, A57

Shanks, T., Metcalfe, N., Chehade, B., et al. 2015, MNRAS, 451, 4238

Shappee, B. J., Stanek, K. Z., Kochanek, C. S., \& Garnavich, P. M. 2017, ApJ, 841,48

Shaw, J. R., Bridges, M., \& Hobson, M. P. 2007, MNRAS, 378, 1365

Silverman, J. M., Ganeshalingam, M., Cenko, S. B., et al. 2012, ApJ, 756, L7

Sim, S. A., Röpke, F. K., Hillebrandt, W., et al. 2010, ApJ, 714, L52

Sim, S. A., Fink, M., Kromer, M., et al. 2012, MNRAS, 420, 3003

Spyromilio, J., Gilmozzi, R., Sollerman, J., et al. 2004, A\&A, 426, 547

Storey, P. J., \& Sochi, T. 2016, MNRAS, 459, 2558

Storey, P. J., Zeippen, C. J., \& Sochi, T. 2016, MNRAS, 456, 1974

Taubenberger, S. 2017, Handbook of Supernovae, ed. A. W. Alsabti \& P. Murdin, 317

Turatto, M., Benetti, S., Cappellaro, E., et al. 1996, MNRAS, 283, 1

Varani, G. F., Meikle, W. P. S., Spyromilio, J., \& Allen, D. A. 1990, MNRAS, 245,570

Woosley, S. E., Pinto, P. A., \& Hartmann, D. 1989, ApJ, 346, 395

Woosley, S. E., Kasen, D., Blinnikov, S., \& Sorokina, E. 2007, ApJ, 662, 487 